\title{
Can a combination of two neuropsychological tests screen for mild neurocognitive disorder better than each test alone? A cross-sectional study
}

Reem Mohamed Sabry ELbedewy ${ }^{* \dagger}$ and Mohamed ELOKL ${ }^{\dagger}$

\begin{abstract}
Background: Early symptoms of dementia may not be apparent and are sometimes even concealed during short office visits initiated for other complaints. The aim of the study is to find out if the combined use of VF/CDT, VF/ BNT, or CDT/BNT could improve the accuracy of detecting mild NCD in an outpatient setting, compared with either test used alone.

Participants: Community-dwelling older adults, attending the outpatient Geriatrics Clinic at Ain Shams University hospitals between June 1, 2017 and January 31, 2018. All participants received a comprehensive geriatric assessment (CGA) which included the mini-mental state examination test. Participants with a score of less than 24 and fulfil DSM5 criteria for mild neurocognitive disorder (NCD) are considered cognitively impaired otherwise are considered normal. Then participants were further examined by the Arabic versions of CDT, BNT, and VF animal category.

Results: We recruited 143 male and female participants mean age $67.17 \pm 5.41$, females are $56.6 \%$, and $48.9 \%$ of all participants have mild NCD according to DSM5 criteria. AUC for individual neurocognitive tests in illiterates is 0.893 for clock drawing test, 0.907 for verbal fluency animal category, and 0.904 for Boston naming test, while AUC for neurocognitive test combinations in illiterates is 0.932 for $V F+C D T, 0.917$ for VF + BNT, and 0.932 for BNT + CDT. On the other hand, AUC for individual neurocognitive tests in educated participants is 0.925 for clock drawing test, 0.921 for verbal fluency animal category, and 0.907 for Boston naming test, whereas AUC for neurocognitive test combinations in educated participants is 0.958 for VF + CDT, 0.963 for VF + BNT, and 0.953 for BNT + CDT.
\end{abstract}

Conclusion: From the current study, we can conclude that any of the studied combinations have better diagnostic accuracy (although small) than individual test in both literate and illiterate older adults.

Keywords: Neuropsychological tests, Verbal fluency, Clock drawing test, Boston naming test, Neurocognitive disorder, Arabic CERAD, Egypt, Elderly, Dementia

\footnotetext{
*Correspondence: reem_sabry@med.asu.edu.eg

${ }^{+}$Reem Mohamed Sabry ELbedewy and Mohamed ELOKL contributed equally to this work.

Department of Geriatrics and Gerontology, Faculty of Medicine, Ain Shams University, Cairo, Egypt
}

\section{SpringerOpen}

(c) The Author(s). 2020 Open Access This article is licensed under a Creative Commons Attribution 4.0 International License which permits use, sharing, adaptation, distribution and reproduction in any medium or format, as long as you give appropriate credit to the original author(s) and the source, provide a link to the Creative Commons licence, and indicate if changes were made. The images or other third party material in this article are included in the article's Creative Commons licence, unless indicated otherwise in a credit line to the material. If material is not included in the article's Creative Commons licence and your intended use is not permitted by statutory regulation or exceeds the permitted use, you will need to obtain permission directly from the copyright holder. To view a copy of this licence, visit http://creativecommons.org/licenses/by/4.0/. 


\section{Background}

The early symptoms of dementia may not be apparent and are sometimes even concealed during short office visits initiated for other complaints. As a consequence, a substantial proportion of participants with dementia remain undiagnosed until later stages [1]. There are different ways for assessment of cognitive function either through single neuropsychological tests or by more detailed comprehensive neuropsychological packages. Assessment of cognitive function as part of the comprehensive geriatric assessment can take a long time in an outpatient clinic setting. Tests like mini-mental state examination (MMSE) and similar tests can take up to $10 \mathrm{~min}$, while brief tests like Mini-Cog takes about 4 $\min$.

One of the comprehensive neuropsychological packages for assessment of cognitive function is The Consortium to Establish a Registry for Alzheimer's disease (CERAD) which has been translated into Arabic and adapted and validated for the Egyptian language and culture [2]. Indeed, CERAD includes a group of simple and quick to administer tests like the animal category test of semantic verbal fluency (VF) [3], the fifteen-item short version of the Boston naming test (BNT) [4], and the clock drawing test (CDT) [5] which can be used alone or in different combinations for detecting of cognitive impairment in an outpatient setting

Diehl and colleagues compared CERAD data between patients with frontotemporal dementia, semantic dementia, and Alzheimer's disease. They found that in the differentiation between frontotemporal dementia and Alzheimer's disease, the combination of animal fluency and Boston naming test correctly classified $90.5 \%$ of patients. While in segregating semantic dementia and Alzheimer's disease, the combination of Boston naming test and mini-mental state examination resulted in correct classification of $96.3 \%$. They concluded that their findings demonstrate that the mini-mental state examination and the language subtests of the CERAD are valuable clinical instruments for the differential diagnosis between early frontotemporal dementia, semantic dementia, and Alzheimer's disease [6].

Knafelc et al. investigated the use of cognitive test and informed report questionnaire to find out whether their combined use could improve the accuracy of detecting dementia in a memory clinic, compared with either test used alone. They used the mini-mental state examination and the short form of the Informant Questionnaire on Cognitive Decline in the Elderly was used. A method of combining the test scores in 323 patients assessed at a memory clinic. They concluded that the combination of cognitive testing and an informant report according to a weighted sum rule did not result in any advantage over the use of the mini-mental state examination alone [7].
The importance of verbal fluency tasks as a cornerstone in cognitive assessment is now well acknowledged, as they provide fast, reliable tools for assessment of both verbal and executive function abilities. This was studied by Farghaly et al. They investigated the effect of age and education on verbal fluency and developed a verbal fluency task that is culture-oriented and non-educationbased to overcome the problem of illiteracy in Egypt. They studied two groups of participants, a normal cognition control group $(n=79)$ and a clinically demented group $(n=32)$, and found that animal, vegetable, and name fluency tasks (unlike phonemic fluency and fruit) were not related to age and education, and they had better criterion validity (area under the receiver operating characteristic curve $[\mathrm{AUC}]=0.96,0.91$, and 0.92, respectively) than did letters and fruits (AUC $=0.74$ and 0.86 , respectively). They suggested cutoff points of 11 for the animal fluency task (sensitivity $=94 \%$, specificity $=93 \%$ ), 11 for vegetables (sensitivity $=84 \%$, specificity $=$ $88 \%$ ), and 18 for names (sensitivity $=91 \%$, specificity $=$ $82 \%)$. They concluded that animals, vegetables, and names are reliable and appropriate categories to be used for culture-oriented and non-education-based verbal fluency tests [8].

There are different ways for assessment of cognitive function either through single neuropsychological tests or by more detailed comprehensive neuropsychological packages. Assessment of cognitive function as part of the comprehensive geriatric assessment can take a long time in an outpatient clinic setting. Therefore, there is an obvious need for valid and less time-consuming cognitive screening tests for accurate and rapid detection of cognitive disorders in older adults. We hypothesized that the combination of these instruments may yield higher accuracy in the identification of cognitively impaired participants than each test alone.

\section{Aim}

To find out if the combined use of VF/CDT, VF/BNT, or $\mathrm{CDT} / \mathrm{BNT}$ could improve the accuracy of detecting mild NCD in an outpatient setting, compared with either test used alone

\section{Methods \\ Study type: a cross-sectional study Participants}

We recruited community-dwelling participants over a period of 8 months starting June 1, 2017 till January 31, 2018, and were continuously enrolled for cognitive evaluation. The sample size was calculated at a confidence level of $95 \%$ and $P$ value of 0.05 . We assumed a response and eligibility rates for all participants at $85 \%$ and study power at 0.8 . The calculated sample size was 
142. We sat clear inclusion and exclusion criteria as follows. Inclusion criteria

1) Age 60 years and above (older adult in this study is operationally defined as 60 and above).

2) Attending the outpatient clinic of Geriatric and Gerontology Department at Ain Shams University hospitals during the period June 1, 2017 to January 31, 2018

3) Able to stay for further assessment

4) Consent to participate in the assessment

5) Both males and females are included.

6) Both literate and illiterate participants are included. (Participants who are unable to read and write and received less than 6 years of formal education were operationally considered illiterate.)

\section{Exclusion criteria}

1. Refused to take part or to continue the full assessment

2. Patients with major neurocognitive disorder or delirium

3. History of another mental disorder or on treatment with psychotropic medications

Patients and next of kin were provided with detailed information regarding the study and the type of assessment. All patients were given equal opportunity to take part or decline participation and all were examined and treated in an equal way. Attendees who fulfilled eligibility criteria were invited to participate, excluding those who declined to take part and withdraw at any stage. Out of 225 patients screened over the study period, we successfully recruited 143 participants who represented the study population.

\section{Methods}

\section{Assessment of the cognitive function}

Cognitive function assessment was done through comprehensive geriatric assessment which included the Arabic version of the MMSE, the DSM-V criteria for neurocognitive disorders, and CDR scale. Accordingly, the 143 patients were divided into group 1 (70 participants) fulfilled the DSM-V criteria for mild neurocognitive disorder and group 2 (73 participants) were without the cognitive disorder.

For the purpose of the study, participants were further assessed by the Arabic versions of CDT, BNT, and VF animal category as part of the Arabic version of the CERAD neuropsychological battery.

1- Verbal fluency: is a neuropsychological test in which participants are asked to produce as many names for animals as possible in $60 \mathrm{~s}$. This category can be semantic, including objects such as animals or fruits, or phonemic, including words beginning with a specified letter, such as p, for example [9]. Verbal fluency tests are used as a measure of executive functions, language, and to evaluate semantic memory. The category fluency test seems to be more sensitive than the phonological test, even during the initial course of Alzheimer's disease, discriminating between early Alzheimer's disease and normal controls. Verbal fluency tests have been validated as brief cognitive assessments for the detection of cognitive impairment and dementia in non-specialist clinical settings $[10,11]$.

2- Boston naming test: is a widely used neuropsychological assessment tool to measure confrontational word retrieval in individuals with aphasia or other language disturbance caused by stroke, Alzheimer's disease, or other dementing disorder [12]. Patients with anomia often have greater difficulties with the naming of not only difficult and low-frequency objects but also easyand high-frequency objects [13].

The BNT stimuli are line drawings of objects with increasing naming difficulty, ranging from simple, highfrequency vocabulary to rare words. Administration requires a spontaneous response within a 20-s period; if such a response is not made, two kinds of prompting cues (one phonemic, one semantic) may be given. Scoring counts the number of spontaneously produced correct responses.

3- Clock drawing test: it is a very quick way to screen a person for possible dementia. It often requires only a minute or two for completion. It does not require much training to administer. It is easier to complete than the MMSE for people with short attention spans. It is freely available and of low cost (costs only pen and paper).

The total score of CDT is 4 and that of $\mathrm{BN}$ is 15 , while that of VF is 10 animal names at least in $1 \mathrm{~min}$.

\section{Statistical method}

The collected data were revised, coded, tabulated, and entered into a PC using the Statistical Package for the Social Sciences (IBM SPSS 10). Descriptive analysis and frequencies generated means and standard deviations for continuous variables and cross tabs for categorical variables. Missing data are deleted list-wise.

For the purpose of the study, we compared those with normal cognitive function with those with mild neurocognitive disorders and participants who are illiterate compared with those with formal education. To study differences between both groups, we conducted chisquare test for gender differences and $t$ test for age, MMSE, CDT, VF, and BNT as continuous variables. For further analysis, we conducted ANOVA with 3 layers included mild neurocognitive disorder versus normal 
cognitive function, gender (males versus females), and education level (literate versus illiterate)

To find out if different combinations of tests would have a better prediction for cognitive disorder compared to a single test, we ran the binary regression analysis (WALD) test for the mild neurocognitive disorder as the dependent variable and different combinations of VF/ $\mathrm{CD} / \mathrm{BN}$ as covariates. This has yielded 3 different probabilities.

1. Probability 1 , verbal fluency and Boston naming

2. Probability 2 , verbal fluency and clock drawing

3. Probability 3 , clock drawing and Boston naming

The 3 outcomes from the binary regression were used to generate ROC, sensitivity, and specificity for $\mathrm{VF} / \mathrm{BN}$, $\mathrm{VF} / \mathrm{CD}$, and $\mathrm{CD} / \mathrm{BN}$ combinations. This was done to all study population and then according to education level.

\section{Results}

The total number of participants recruited is 143, 49\% have a mild neurocognitive disorder $(n=70)$ and $51 \%$ had no neurocognitive disorder and were labeled as normal controls $(n=73)$. Females represented $56.6 \%$ of all participants $(n=81)$ and males were $43.4 \%(n=62)$. Of the male participants, $41.9 \%(n=26)$ have mild NCD, while $54.3 \%$ of females have mild NCD $(n=44)$. Out of the 143 participants, $50.3 \%$ were illiterate $(n=72)$ and the rest have received formal education and were able to read and write.

The age range of participants was 60 to 85 years with a mean age of $67.17 \pm 5.41$ for all participants. For participants with mild NCD, the mean age was $67.73 \pm 5.25$, while that for people without NCD was $66.62 \pm 5.55$ and the difference is statistically significant $(P<0.05)$.

The group with mild NCD have a statistically significantly lower mean $( \pm$ SD) for MMSE, CDT, VF, and BNT compared to the group without NCD. The mean and SD for those tests are as follows: MMSE $17.5 \pm 3.4$, CDT $1.03 \pm 1.02$, VF $8.5 \pm 2.4$, and BNT $9.6 \pm 2.4$ compared to $26.24 \pm 2.07,3.07 \pm 0.93,12.8 \pm 1.78$, and $13.3 \pm 1.7$ in people without NCD respectively. This is presented in Table 1 together with demographic data where chi-square test was used for gender differences between the 2 groups with Pearson's chi square of 2.15 , and $t$ test was used for the other continuous variables including age, MMSE, CDT, VF, and BNT.

The performance in the neurocognitive tests according to education level is presented in Table 2. We conducted ANOVA with 3 layers including mild neurocognitive disorder versus normal cognitive function, gender (males versus females), and education level (literate versus illiterate). As shown in Table 2, comparing illiterates to those with formal education, the $P$ value is above 0.05 for age, MMSE, VF, and CDT, indicating no statistically significant difference between the groups and is less than 0.05 for Boston naming only.

Table 3 shows the binary logistic regression analysis for different probabilities of combined tests for all participants. The table also presents the AUC for different combinations in all study population. Table 4 shows the validity of different combination of neuropsychological test and the positive and negative likelihood of different combination in detecting mild NCD in all participants, while Table 5 shows the sensitivity and specificity according to education level in both single tests and different combinations. Finally, Table 6 shows AUC for single and combined tests in illiterate and literate participants.

Table 5 shows the sensitivity of individual neurocognitive tests in the illiterate group which is $0.971,0.941$, and 0.853 for CDT, VF, and BNT respectively, while specificity for those tests in illiterates is $0.316,0.579$, and 0.211 for CDT, VF, and BNT respectively. The sensitivity of neurocognitive test combinations in illiterate group is 0.971, 0.941, and 0.971 for VF + CDT, VF + BNT, and BNT + CDT respectively, and the specificity of those combinations in illiterates is $0.632,0.605,0.421$ for VF + $\mathrm{CDT}, \mathrm{VF}+\mathrm{BNT}$, and $\mathrm{BNT}+\mathrm{CDT}$ respectively.

On the other side, the sensitivity of individual neurocognitive tests in the educated group is $0.944,0.972$, and

Table 1 Demographic and cognitive variables of all participants

\begin{tabular}{llll}
\hline & Mild NCD, 49\% $(n=70)$ & Normal CF, 51\% $(n=73)$ & $\boldsymbol{P}$ value \\
\hline *Male (62) & $41.9 \%(n=26)$ & $58.1 \%(n=36)$ & $\mathbf{0 . 0 5}$ \\
*Female (81) & $54.3 \%(n=44)$ & $45.7 \%(n=37)$ & $\mathbf{0 . 0 4}$ \\
Age & $67.73 \pm 5.25$ & $66.62 \pm 5.55$ & $\mathbf{0 . 0 4}$ \\
MMSE & $17.51 \pm 3.42$ & $26.25 \pm 2.07$ & $\mathbf{0 . 0 0 1}$ \\
CDT & $1.03 \pm 1.02$ & $3.07 \pm 0.93$ & $\mathbf{0 . 0 0 1}$ \\
VF & $8.56 \pm 2.41$ & $12.88 \pm 1.78$ & $\mathbf{0 . 0 0 1}$ \\
BNT & $9.64 \pm 2.47$ & $13.37 \pm 1.62$ & $\mathbf{0 . 0 0 1}$ \\
\hline
\end{tabular}

MMSE mini-mental state examination test, CDT clock drawing test, VF verbal fluency, BNT Boston naming test, NCD neurocognitive disorder, CF cognitive function ${ }^{*}$ Chi-square test and Pearson's chi-square $=2.15$

$P$ value more than 0.05 is not significant 
Table 2 Performance in neurocognitive tools according to education level

\begin{tabular}{|c|c|c|c|c|c|c|c|c|c|c|}
\hline \multicolumn{5}{|c|}{ Illiterate, 50.3\% $(\boldsymbol{n}=72)$} & \multicolumn{4}{|c|}{ Any formal education, $49.7 \%(\boldsymbol{n}=71)$} & \multirow[t]{2}{*}{${ }^{*} f$} & \multirow{2}{*}{$\begin{array}{l}{ }^{* * P} P \\
\text { value }\end{array}$} \\
\hline & \multicolumn{2}{|c|}{ Male, $36 \%(n=26)$} & \multicolumn{2}{|c|}{ Female, $64 \%(n=46)$} & \multicolumn{2}{|c|}{ Male, $51 \%(n=36)$} & \multicolumn{2}{|c|}{ Female, $49 \%(n=35)$} & & \\
\hline & $\begin{array}{l}\text { Mild NCD, } \\
39 \%(n= \\
10)\end{array}$ & $\begin{array}{c}\text { No cog. } \\
\text { impairment, } \\
61 \%(n=16)\end{array}$ & $\begin{array}{c}\text { Mild NCD, } \\
52 \%(n= \\
24)\end{array}$ & $\begin{array}{c}\text { No cog. } \\
\text { impairment, } \\
48 \%(n=22)\end{array}$ & $\begin{array}{c}\text { Mild NCD, } \\
44 \%(n= \\
16)\end{array}$ & $\begin{array}{c}\text { No cog. } \\
\text { impairment, } \\
56 \%(n=20)\end{array}$ & $\begin{array}{l}\text { Mild NCD, } \\
57 \%(n= \\
20)\end{array}$ & $\begin{array}{c}\text { No cog. } \\
\text { impairment, } \\
43 \%(n=15)\end{array}$ & & \\
\hline Age & $66.3 \pm 3.92$ & $67.38 \pm 6.0$ & $\begin{array}{l}70.42 \pm \\
6.01\end{array}$ & $66.14 \pm 4.61$ & $\begin{array}{l}67.62 \pm \\
5.38\end{array}$ & $67.15 \pm 5.37$ & $\begin{array}{l}65.30 \pm \\
3.06\end{array}$ & $65.80 \pm 6.75$ & 1.05 & $\begin{array}{l}> \\
0.05\end{array}$ \\
\hline MMSE & $17.7 \pm 2.87$ & $26.12 \pm 2.09$ & $\begin{array}{l}16.04 \pm \\
3.63\end{array}$ & $25.09 \pm 1.11$ & $18.0 \pm 4.07$ & $27.45 \pm 2.06$ & $18.8 \pm 2.19$ & $26.47 \pm 2.36$ & 1.3 & $\begin{array}{l}>05 \\
0.05\end{array}$ \\
\hline$C D$ & $0.9 \pm 0.88$ & $3 \pm 1.03$ & $1.04 \pm 0.81$ & $2.09 \pm 1.01$ & $0.81 \pm 1.22$ & $3.45 \pm 0.81$ & $1.25 \pm 1.16$ & $3.33 \pm 0.72$ & 1.7 & $\begin{array}{l}> \\
0.05\end{array}$ \\
\hline VF & $8.06 \pm 2.71$ & $12.6 \pm 1.63$ & $8.29 \pm 2.79$ & $13.09 \pm 1.66$ & $8.13 \pm 1.67$ & $12.65 \pm 2.25$ & $9.50 \pm 2.16$ & $13.13 \pm 1.46$ & 1.06 & $\begin{array}{l}> \\
0.05\end{array}$ \\
\hline BN & $9.20 \pm 2.25$ & $12.7 \pm 2.29$ & $8.79 \pm 2.84$ & $13.23 \pm 1.34$ & $\begin{array}{l}10.12 \pm \\
1.63\end{array}$ & $13.65 \pm 1.42$ & $\begin{array}{l}10.50 \pm \\
2.44\end{array}$ & $13.87 \pm 1.25$ & 3.9 & $\begin{array}{l}< \\
0.05\end{array}$ \\
\hline
\end{tabular}

MMSE mini-mental state examination test, $C D T$ clock drawing test, $V F$ verbal fluency, $B N T$ Boston naming test, $N C D$ neurocognitive disorder, $C F$ cognitive function *ANOVA $f$ value

** $P$ value more than 0.05 is not significant

0.917 for CDT, VF, and BNT respectively, while specificity for those tests in the educated group is $0.457,0.343$, and 0.457 for CDT, VF, and BNT respectively. The sensitivity of neurocognitive test combinations in educated group is $0.972,0.972$, and 0.972 for $\mathrm{VF}+\mathrm{CDT}$, VF + $\mathrm{BNT}$, and BNT + CDT respectively, while specificity for those combinations in educated group is 0.543, 0.629, and 0.543 for VF + CDT, VF + BNT, and BNT + CDT respectively.

Accordingly, in the educated group, the sensitivity of BNT + CDT is better than BNT or CDT individually but equal to the sensitivity of VF alone. Specificity, in the educated group, for VF + CDT is better than the specificity of VF or CDT individually and specificity for VF + BNT is better than VF or BNT individually. The specificity of $\mathrm{BNT}+\mathrm{CDT}$ is better than the specificity of BNT or CDT individually. Obviously, there is a better specificity with combination VF + BNT in the educated group.

The AUC for individual neurocognitive tests in illiterates is $0.893,0.907$, and 0.904 for CDT, VF animal category, and BNT respectively, while that for neurocognitive test combinations in illiterates is 0.932 , 0.917, and 0.932 for VF + CDT, VF + BNT, and BNT + CDT respectively as shown in Fig. 1.

Table 3 Binary logistic regression analysis for different probabilities of combined tests

\begin{tabular}{llllll}
\hline & B & S.E. & Wald & Sig. & AUC \\
\hline VF/BN & -0.556 & 0.215 & 6.698 & $\mathbf{0 . 0 1 0}$ & 0.940 \\
CD/NF & -1.957 & 0.585 & 11.192 & $\mathbf{0 . 0 0 1}$ & 0.970 \\
CD/BN & -2.629 & 0.712 & 13.648 & $\mathbf{0 . 0 0 0}$ & 0.960 \\
\hline
\end{tabular}

Wald $\left(t^{2}\right)$ chi square distributed with $\mathrm{df}=1$
On the other hand, the AUC for individual neurocognitive tests in educated subjects is as follows: 0.925, 0.921, and 0.907 for CDT, VF animal category, and BNT respectively, whereas that for neurocognitive test combinations in educated subjects is $0.958,0.963$, and 0.953 for $\mathrm{VF}+\mathrm{CDT}, \mathrm{VF}+\mathrm{BNT}$, and $\mathrm{BNT}+\mathrm{CDT}$ respectively as shown in Fig. 2.

Collectively, for illiterates, the best AUC, sensitivity, and specificity are that of the combination of $\mathrm{VF}+\mathrm{CDT}$, while for educated is that of the combination of $\mathrm{VF}+$ BNT.

Figures 1 and 2 show ROC curve and specificity and sensitivity for different neurocognitive test combinations in illiterate and educated participants respectively.

\section{Discussion}

The older adults may have a mild neurocognitive disorder that can progress to dementia with the devastating effects and consequences of dementia on patient, family, and even the whole society. The screening for cognitive disorders in outpatient clinics is an essential part of the comprehensive geriatric assessment (CGA) which is time-consuming. Accordingly, there is a need for short, accurate, and easy to administer neuropsychological tests to pick up cases with cognitive impairment.

This cross-sectional study includes 143 participants recruited over 8 months from outpatient Geriatric Medicine clinic at Faculty of Medicine, Ain Shams University hospitals, Cairo, Egypt. We included males and females with age range 60 to 85 years (mean and SD $67.17 \pm$ 5.41) and almost half of participants have mild NCD according to DSM-V criteria and the rest had a normal cognitive function. 
Table 4 Validity of different combinations of neuropsychological test for all participants

\begin{tabular}{lllll}
\hline Combination & Sensitivity & Specificity & Positive likelihood & Negative likelihood \\
\hline VF/BNT & $0.91(\mathrm{Cl}, 0.84-0.96)$ & $0.61(\mathrm{Cl}, 0.48-0.73)$ & $2.37(\mathrm{Cl}, 1.72-3.26)$ & $7.43(\mathrm{Cl}, 3.71-14.85)$ \\
VF/CDT & $0.90(\mathrm{Cl}, 0.83-0.95)$ & $0.451(\mathrm{Cl}, 0.32-0.58)$ & $1.65(\mathrm{Cl}, 1.30-2.09)$ & $4.86(\mathrm{Cl}, 2.46-9.60)$ \\
CDT/ BNT & $0.98(\mathrm{Cl}, 0.94-0.99)$ & $0.32(\mathrm{Cl}, 0.20-0.45)$ & $1.46(\mathrm{Cl}, 1.22-1.73)$ & $13.29(\mathrm{Cl}, 4.30-22.29)$ \\
\hline
\end{tabular}

$\mathrm{Cl}$ confidence interval at $95 \%$

The known verbal fluency animal category test (VF), Boston naming test (BNT) short form, and clock drawing test (CDT) are brief tests for cognitive screening and they are translated, adapted, and validated in the Arabic language. In the current study, we tested the diagnostic performance of different combinations of those tests in comparison to each individual test.

The area under the curve for any of the studied combination is better than any individual test in both educated and illiterate participants. Individual tests and test combinations have higher sensitivity and low specificity in both groups. It was also noted that the combination of VF and BNT has better sensitivity and specificity in the educated group, while that was better with VF/CDT in the illiterate group. In a study by de Noronha et al. to determine the influence of education on the performance of healthy adults on the CDT found that CDT was not strongly influenced by education in its application, except for illiterate individuals, who attained statistically significant lower scores [14]. Accordingly, it can be used clinically for screening cognitive deficits irrespective of education. The absence of difference among the groups exposed to formal education on comparison of performance on the CDT according to different educational levels suggests that the CDT can be used as a cognitive screening task even in patients with low education.

Ladeira et al. evaluated combining cognitive screening tests for the evaluation of mild cognitive impairment in the elderly. They examined cognitive function in 247 older adults in Brazil. Their results showed that neither test alone proved adequate for the correct separation of patients with mild cognitive impairment from healthy subjects and combining of the mini-mental state examination with the verbal fluency test and clock drawing test did not improve diagnostic accuracy, as compared

Table 5 Sensitivity and specificity according to education level

\begin{tabular}{lllllll}
\hline & CDT & VF & BN & VF/CDT & VF/BNT & BNT/CDT \\
\hline Illiterate & & & & & & \\
Sensitivity & 0.971 & 0.941 & 0.853 & 0.971 & 0.941 & 0.971 \\
$\begin{array}{c}\text { Specificity } \\
\text { Literate }\end{array}$ & 0.316 & 0.579 & 0.211 & 0.632 & 0.605 & 0.421 \\
Sensitivity & 0.944 & 0.972 & 0.917 & 0.972 & 0.972 & 0.972 \\
Specificity & 0.457 & 0.343 & 0.457 & 0.543 & 0.629 & 0.543 \\
\hline
\end{tabular}

to the mini-mental state examination alone, in the identification of patients with mild cognitive impairment or Alzheimer's disease. And they concluded that the data do not support the notion that the combination of test scores is better than the use of mini-mental state examination scores alone in the screening for Alzheimer's disease. In our study, we have not combined test score, we rather did a binary regression analysis generated 3 probabilities which then was used to generate AUC. We have not used MMSE in any of the combinations and our aim was also different in terms of finding short reliable and valid test to be easily used in an outpatient setting [15].

In a recent study, Payton and colleagues investigated the extent to which combining cognitive markers increases the predictive value for future dementia when compared to individual markers. As part of the Swedish National Study on Aging and Care, they performed a neuropsychological assessment for 2357 participants (60 + years) without dementia, and found that category fluency was the strongest individual predictor of dementia 6 years before diagnosis [area under the curve $(\mathrm{AUC})=$ .903]. The final model included tests of verbal fluency, episodic memory, and perceptual speed (AUC $=.913$ ); these three domains were found to be the most predictive across a range of different subgroups. They concluded that combining markers from different cognitive domains leads to increased accuracy in predicting future dementia 6 years later. Markers from the verbal fluency, episodic memory, and perceptual speed domains consistently showed high predictively across subgroups stratified by age, sex, education, apolipoprotein $\mathrm{E} \in 4$ status, and dementia type [16].

As a cross-sectional study, we can prove our assumption regarding the use of those accessible neurocognitive tests in different combinations; however, the limitations of the study included the limited number of older adults included and the lack of follow-up and repeated assessment. From the current study, we can conclude that any

Table 6 AUC for single and combined tests in illiterate and literate participants

\begin{tabular}{lllllll}
\hline & CDT & VF & BN & VF/CDT & VF/BNT & BNT/CDT \\
\hline AUC illiterate & 0.893 & 0.907 & 0.904 & 0.932 & 0.917 & 0.932 \\
AUC literate & 0.925 & 0.921 & 0.907 & 0.958 & 0.963 & 0.953 \\
\hline
\end{tabular}




\section{ROC Curve}

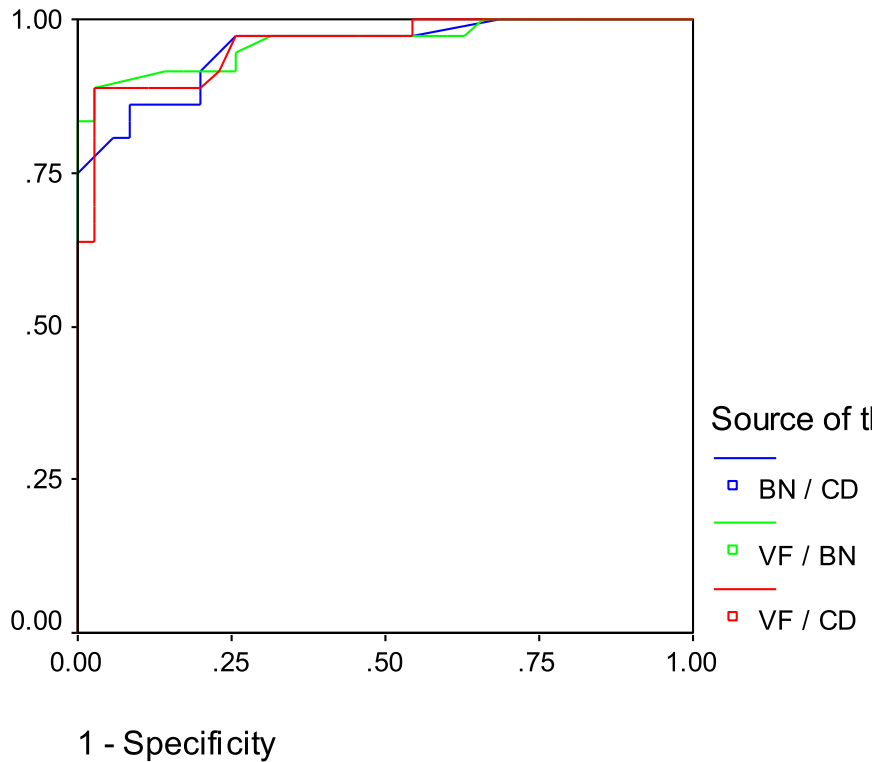

Fig. 1 ROC for different neurocognitive test combinations in illiterates

of the studied combinations have better diagnostic accuracy (although small) than individual test whether in educated or illiterate older adults. So, despite the high performance of any of studied tests when used alone, it is better, whenever convenient, in busy outpatient clinics to use the best combination of the studied neuropsychological tests according to education as combinations can improve detection of mild NCD and help to exclude participants without mild NCD. Such tests in combination can be administered in a few minutes and they are handy and easy to apply.

\section{ROC Curve}

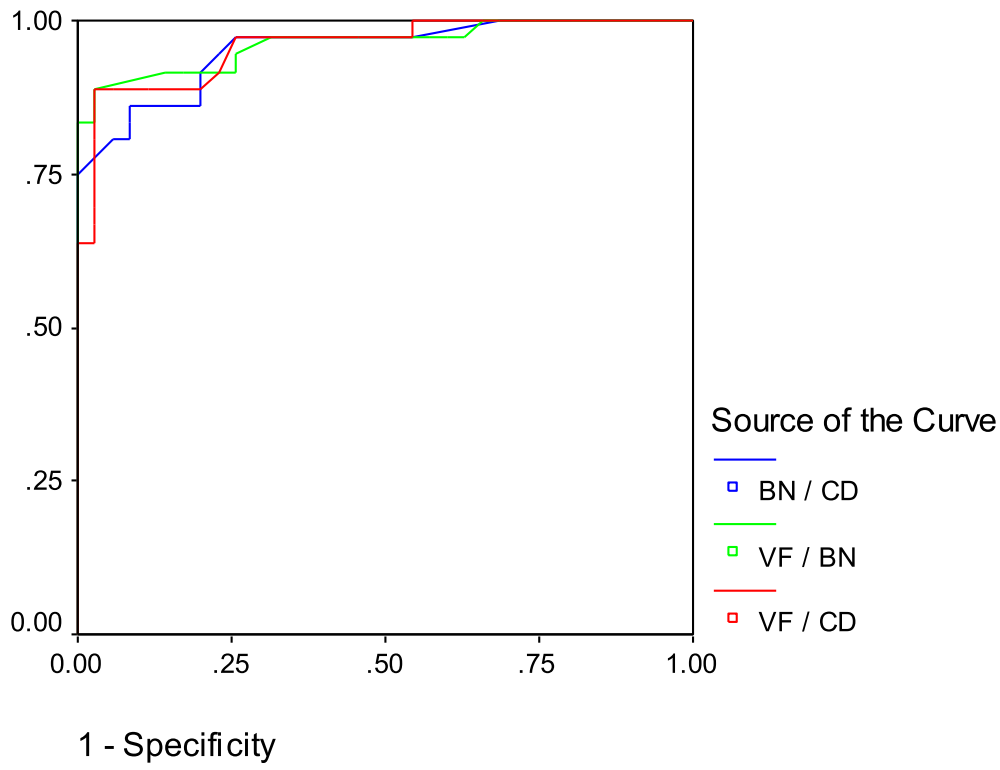

Fig. 2 ROC for different neurocognitive test combinations in educated subjects 
The differences between VF/CD and VF/BN are not significantly high between illiterates and educated group; hence, we find it more appropriate to recommend adding $\mathrm{CD}$ to VF than adding BN. All needed is a sheet of paper and a pen/pencil which can be found in outpatient clinics. We would encourage using VF and CD as quick valid tools for assessment of cognitive functions in outpatient clinics. We can call this test model v.Clock (verbal fluency combined with clock drawing) and recommend testing it in a larger study.

\section{Conclusion}

From the current study, we can conclude that any of the studied combinations have better diagnostic accuracy (although small) than individual test in both literate and illiterate older adults. The use of verbal fluency and clock drawing combination (v.Clock) may be more appropriate for use in the outpatient setting.

\section{Recommendations}

Further studies are needed on a larger number of older adults using v.Clock model.

\section{Abbreviations}

CGA: Comprehensive geriatric assessment; MMSE: Mini-mental state examination; CERAD: The Consortium to Establish a Registry for Alzheimer's disease; CDR: Clinical Dementia Rating Scale; DSM V: Diagnostic and Statistical Manual of Mental Disorders-5; VF: Verbal fluency; BNT: Boston naming test; CDT: Clock drawing test; NCD: Neurocognitive disorder; SPSS: Statistical Package for the Social Sciences; ROC: Receiver operating characteristic; AUC: The area under the curve; SD: Standard deviation

\section{Acknowledgements}

Not applicable.

\section{Authors' contributions}

Both authors, R E and M E, equally participated in the conception, design of the work, data collection, and interpretation of the data, review of the results' analysis, writing, revising, and editing this manuscript. Both authors read and approved the final submitted manuscript.

\section{Authors' information}

Both authors are associate professors at Geriatrics and Gerontology department, Faculty of Medicine, Ain Shams University, Cairo, Egypt.

Dr Mohammed ELOKL is currently working as Consultant Geriatric Medicine at University Hospitals Bristol and Weston NHS Foundation Trust, England, UK.

\section{Funding}

"Not applicable."

\section{Availability of data and materials}

"The data generated and analyzed during this study are included in this article and available from the corresponding author on further reasonable request."

\section{Competing interests}

"The authors declare that they have no competing interests."

Received: 4 December 2019 Accepted: 21 July 2020

Published online: 15 October 2020

\section{References}

1. Seematter-Bagnoud and Büla (2018) Public Health Reviews $39: 8$ https://doi. org/10.1186/s40985-018-0086-7

2. Elokl M., Elbanouby M., Mortagy M., elAtrebi M., ELsabwa M (2001) Ain shams university, faculty of medicine department of Geriatrics. Prevalence of $A D$ and other types of dementia in Egypt. International Psychogeriatric, volume 13, Supplement2, 2001.

3. Benton AL (1968) Differential behavioural effects in frontal lobe disease. Neuropsychologia 6:53-60

4. Kaplan EF, Goodglass H, Weintraub S (1978) The Boston naming test. MA, Veterans Administration Medical Center, Boston

5. Freedman MI, Leach L, Kaplan E, Winocur G, Shulman KJ, Delis DC (eds) (1994) Clock drawing. Oxford University Press, Oxford

6. J. Diehl, A. U. Monsch, C. Aebi, S. Wagenpfeil, S. Krapp, T. Grimmer, W. Seeley, H. Förstl, A. Kurz. Frontotemporal dementia, semantic dementia, and Alzheimer's disease (2005): the contribution of standard neuropsychological tests to differential diagnosis. Journal of Geriatric Psychiatry and Neurology / Vol. 18, No. 1.

7. Knafelc R, Lo Giudice D, Harrigan S, Cook R, Flicker L, Mackinnon A, Ames D (2003) The combination of cognitive testing and an informant questionnaire in screening for dementia. Age Ageing. 32(5):541-547

8. Farghaly M, Hussein M, Hassan A, Hegazy M, Sabbah A (2018 Sep) 2018. Testing of verbal fluency in Egyptians: cultural and educational challenges. Cogn Behav Neurol. 31(3):133-141

9. Lezak, Muriel Deutsch (1995) Neuropsychological assessment. Oxford [Oxfordshire]: Oxford University Press. ISBN 0-19-509031-4.

10. Sebaldt R, Dalziel W, Massoud F, Tanguay A, Ward R, Thabane L, Melnyk P, Landry P-A, Lescrauwaet B (2009) "Detection of cognitive impairment and dementia using the animal fluency test: the DECIDE study". The Canadian Journal of Neurological Sciences. Le Journal Canadien Des Sciences Neurologiques. 36(5):599-604

11. Canning, S. J. Duff; Leach, L.; Stuss, D.; Ngo, L.; Black, S. E. (2004-02-24) "Diagnostic utility of abbreviated fluency measures in Alzheimer disease and vascular dementia". Neurology. 62 (4): 556-562.

12. del Toro CM, Bislick LP, Comer M, Velozo C, Romero S, Rothi G, Leslie J, Kendall DL (2010) Development of a short form of the Boston naming test for individuals with aphasia. Journal of Speech, Language, and Hearing Research. 54(4):1089-1100

13. Kaplan; Goodglass, Harold; Weintraub, Sandra (1983) Boston naming test. Philadelphia: Lea \& Febiger. OCLC 10450471.[non-primary source needed]

14. de Noronha IFC, Barreto SDS, Ortiz KZ (2018) The influence of education on performance of adults on the clock drawing test. Dement Neuropsychol 12(1):61-67

15. Ladeira RB, Diniz BS, Nunes PV, Forlenza OV 2009 Combining cognitive screening tests for the evaluation of mild cognitive impairment in the elderly Clinics (Sao Paulo). 2009;64(10):967-73.

16. Payton NM, Rizzuto D, Fratiglioni L, Kivipelto M, Bäckman L (2020) Laukka EJ Combining cognitive markers to identify individuals at increased dementia risk: influence of modifying factors and time to diagnosis. J Int Neuropsychol Soc. 24:1-13

\section{Publisher's Note}

Springer Nature remains neutral with regard to jurisdictional claims in published maps and institutional affiliations.

\section{Ethics approval and consent to participate}

The study was approved by the ethics committee of the Faculty of Medicine Ain Shams University, Cairo, Egypt "Reference Number is not available." Informed oral consent to participate in the study was received from each participant and/or his/her next of kin. Patients and/or their next of kin who declined to participate were excluded, as were those who refused to complete the assessment. 